\title{
Modulation of Inflammatory Response and Induction of Bone Formation Based on Combinatorial Effects of Resveratrol
}

\author{
Katy E Rutledge ${ }^{1}$, Qingsu Cheng ${ }^{2}$ and Ehsan Jabbarzadeh ${ }^{1,2,3 *}$
}

${ }^{1}$ Department of Chemical Engineering, University of South Carolina, Columbia, SC, 29208, USA

${ }^{2}$ Biomedical Engineering Program, University of South Carolina, Columbia, SC, 29208, USA

${ }^{3}$ Department of Orthopaedic Surgery, University of South Carolina School of Medicine, Columbia, SC, 29209, USA

\begin{abstract}
The success of bone tissue engineering strategies critically depends on the rapid formation of a mature vascular network in the scaffolds after implantation. Conventional methods to accelerate the infiltration of host vasculature into the scaffolds need to consider the role of host response in regulation of bone tissue ingrowth and extent of vascularization. The long term goal of this study was to harness the potential of inflammatory response to enhance angiogenesis and bone formation in three dimensional (3D) scaffolds. Towards this goal, we explored the use of resveratrol, a natural compound commonly used in complementary medicine, to enable the concurrently (i) mediate M1 to M2 macrophage plasticity, (ii) impart natural release of angiogenic factors by macrophages and (iii) enhance osteogenic differentiation of human mesenchymal stem cells (hMSCs). We mapped the time-dependent response of macrophage gene expression as well as hMSC osteogenic differentiation to varying doses of resveratrol. The utility of this approach was evaluated in 3D poly (lactide-co-glycolide) (PLGA) sintered microsphere scaffolds for bone tissue engineering applications. Our results altogether delineate the potential to synergistically accelerate angiogenic factor release and upregulate osteogenic signaling pathways by "dialing" the appropriate degree of resveratrol release.
\end{abstract}

Keywords: Resveratrol; Mesenchymal stem cells; Macrophage polarization; Inflammatory microenvironment; Osseointegration

\section{Introduction}

Tissue engineering offers a revolutionary approach to restore critical-size defects resulting from trauma, infection, tumor resection or other musculoskeletal diseases [1]. This is traditionally accomplished by utilizing a combination of biomaterials, cells, and signaling factors. Biomaterials provide a three-dimensional (3D) substrate with specific engineered characteristics for cells to attach and proliferate. Growth factors supply essential signaling cues for cells to migrate and differentiate into the desired tissue type. Cells seeded on 3D biomaterials contribute to the healing process through signaling events that guide newly formed tissue integration with the host tissue.

The success of tissue engineering strategies is contingent on the ability of blood vessels to form within the scaffolds and supply nutrients to the transplanted cells [2]. This process is controlled through a cascade of events that are mediated by host inflammatory response. Inflammation is the process by which the body protects itself from intruders, and if left uncontrolled, potentially interferes with the integration of implanted biomaterials. The complicated interplay of cells and cytokines at the interface of an implanted biomaterial must be deconstructed in order to properly design an approach to harness inflammation and promote wound healing.

The host inflammatory response is commonly initiated through the release of serum proteins from the vasculature binding to the implanted biomaterial and forming a provisional matrix composed of fibrin, platelet granule components such as thrombospondin, transforming growth factor alpha (TGF- $\alpha$ ), transforming growth factor beta (TGF- $\beta$ ), platelet-derived growth factor (PDGF), platelet factor 4 (PF4), and platelet-derived endothelial cell growth factor (ECGF1) [3]. This process is followed by monocytes exiting the capillaries, migrating to the surface of biomaterials, and differentiating into M1 macrophages. The secretion of cytokines such as tumor necrosis factor alpha (TNF- $\alpha$ ), interleukin-1 beta (IL-1 $\beta$ ), interleukin-6 (IL$6)$, and monocyte chemotactic protein $1(\mathrm{MCP}-1)$ drives the acute inflammation response $[4,5]$. The next event in the host response to an implanted biomaterial is chronic inflammation, which is defined by the presence of macrophages, monocytes, lymphocytes, blood vessel proliferation, and connective tissue formation [6-9]. Foreign body giant cells and macrophages exist at tissue-implant interface for the duration of the biomaterial's lifetime [10-14]. A fibrous capsule forms as a result, separating the biomaterial from the host tissue [3]. Several strategies have been designed to modulate the host inflammation in response to biomaterials [15-23]. In one approach, a hydrophilic boundary between implant and host tissue was created to enhance the biocompatibility of implants. In another approach, anti-inflammatory biomolecules were utilized to inhibit the secretion of mediators such as leukotrienes and prostaglandins, and delay fibroblast capsule formation [24]. A major drawback of these methods is the numerous undesired systemic side effects [25]. For example, anti-inflammatory corticosteroid based drugs inhibit new blood vessel formation necessary for nutrient exchange in the implanted tissue $[26,27]$. Another commonly used method is the induction of angiogenesis based on the use of growth factors [2830]. Despite its success, this method can overexpose tissues to growth factors leading to arthritis and tumor formation [31-33].

Efforts to identify compounds with anti-inflammation properties have led to discovery of resveratrol, a polyphenol found in the skin of grapes [34,35]. Strikingly, this molecule has been shown to exhibit therapeutic effects in a number of diseases including cancer,

*Corresponding author: Ehsan Jabbarzadeh, Orthopaedic Surger University of South Carolina Columbia, SC 29208, USA, Tel: 803-777-3297 Fax: 803-777-8265; E-mail: jabbarza@cec.sc.edu

Received November 24, 2015; Accepted Janaury 12, 2016; Published January 25, 2016

Citation: Rutledge KE, Cheng Q, Jabbarzadeh E (2016) Modulation of Inflammatory Response and Induction of Bone Formation Based on Combinatorial Effects of Resveratrol. J Nanomed Nanotechnol 7: 350. doi:10.4172/2157-7439.1000350

Copyright: (c) 2016 Rutledge KE, et al. This is an open-access article distributed under the terms of the Creative Commons Attribution License, which permits unrestricted use, distribution, and reproduction in any medium, provided the original author and source are credited. 
cardiovascular failure, viral infections, neurodegeneration, and ischemic injuries [36-46]. The exact mechanisms by which resveratrol impacts inflammation is under intense investigation. Suppression of transcription factor nuclear factor kappa B (NF- $\kappa B)$, TNF- $\alpha$ and interleukin-1 (IL-1), prostaglandin E2 (PGE2), and nitric oxide (NO) synthesis have been demonstrated to be potential means by which resveratrol attenuates inflammation [47-49]. Further studies are warranted to explore the signaling cascades governing the antiinflammatory potential of resveratrol in the context of wound healing and implant integration.

In addition to its anti-inflammatory characteristic, resveratrol has been found to possess pro-osteogenic properties [50]. Augmentation of Wnt signaling pathway, activation of sirtuin 1 (SIRT-1), and acetylation of runt-related transcription factor 2 (RUNX2) have been shown to be the underlying mechanism behind the induction of osteogenesis in stem cells by resveratrol [51-54]. Additionally, reports of the bone inductive effects of resveratrol through the activation of estrogen receptor have highlighted this natural compound as a viable candidate factor for bone tissue engineering applications.

The long term objective of this study is to delineate the potential of resveratrol to accelerate the formation of bone and vasculature in tissue engineered scaffolds. The major hypothesis underlying our effort is that resveratrol can be used to simultaneously (1) induce the osteogenic differentiation of hMSCs seeded onto 3D porous scaffolds, and (2) regulate the intricate balance of M1 - M2 macrophage phenotype switch. We tested these hypotheses by assessing the time-dependent effect of varying resveratrol doses on macrophage gene expression as well as hMSCs osteogenic differentiation in 2D and 3D culture systems.

\section{Materials and Methods}

\section{Human monocyte cell culture}

Human THP-1 monocytes (Sigma) were cultured in basal medium consisting of RPMI 1640 (Sigma), 2 mM L-Glutamine (Gibco), 10\% FBS (Atlas), and 1\% penicillin/streptomycin (Invitrogen). Medium was changed every other day, and cells were kept at a density of 3-8 x $10^{6}$ cells $/ \mathrm{mL}$. THP-1 monocytes were seeded in each well of a 24 well plate at a number of 500,000 cells per well. Depending on the experimental conditions, cells were kept at an undifferentiated state, were differentiated to M0 macrophages, or were differentiated to M1 macrophages.

\section{Differentiation of monocytes to macrophages}

THP-1 monocytes were differentiated to M0 macrophages by adding $200 \mathrm{nM}$ phorbol 12-myristate 13-acetate (PMA) to the basal medium for 24 hours after which cells adhered to the tissue culture plate. To differentiate M0 macrophages to M1 macrophages, basal medium supplemented with $1 \mu \mathrm{g} / \mathrm{mL}$ lipopolysaccharide (LPS) (Sigma) and $20 \mathrm{ng} / \mathrm{mL}$ recombinant interferon gamma (IFN $\gamma$ ) (Peprotech) was added to the wells for 24 hours. Differentiation of M0 macrophages to M2 macrophages was accomplished by adding $20 \mathrm{ng} / \mathrm{mL}$ recombinant interleukin 4 (IL-4) (Peprotech) as well as $20 \mathrm{ng} / \mathrm{mL}$ recombinant interleukin-13 (IL-13) (Peprotech) to the basal medium, and then culturing M0 macrophages in the differentiation medium for 18 hours.

\section{Effect of resveratrol on M1 macrophages}

Resveratrol was added to basal THP-1 monocytes culture medium at the concentrations of $1 \mu \mathrm{M}, 10 \mu \mathrm{M}$, and $25 \mu \mathrm{M}$, and gene expression of cytokines VEGF, TNF- $\alpha$, IL-6, macrophage mannose receptor 1
(MRC-1), and interleukin 10 (IL-10) were analyzed using qRT-PCR after resveratrol exposure durations of 2 and 5 days.

The total RNA was extracted and purified from the samples using the GeneJET RNA Purification Kit (Thermo Scientific). Briefly, RNA $(0.5 \mu \mathrm{g})$ was prepared as a template for single-strand cDNA synthesis using the RevertAid First Strand cDNA Synthesis Kit (Thermo Scientific). First, genomic DNA was removed from the RNA sample by applying a mixture of the supplied reaction buffer with $\mathrm{MgCl} 2$, DNase I, and nuclease-free water to a total volume of $10 \mu \mathrm{L}$. The samples were then incubated at $37^{\circ} \mathrm{C}$ for 30 minutes, after which $1 \mu \mathrm{L} 50 \mathrm{mM}$ EDTA was added.

The samples were placed in a $65^{\circ} \mathrm{C}$ water bath for 10 minutes. The template RNA was mixed with $1 \mu \mathrm{L}$ oligo (dT) 18 primer and nuclease-free water to a volume of $12 \mu \mathrm{L}$, followed by the addition of $4 \mu \mathrm{L}$ of $5 \mathrm{X}$ Reaction Buffer, $1 \mu \mathrm{L}$ Ribolock RNase Inhibitor, $2 \mu \mathrm{L} 10$ mM dNTP Mix, and $1 \mu \mathrm{L}$ RevertAid M-MuLV Reverse Transcriptase. This mixture was incubated at $42^{\circ} \mathrm{C}$ for 1 hour in a Bio-Rad CFX96 thermocycler instrument. To detect gene expression, the SensiFAST SYBR No-ROX Kit (Bioline) was used for qPCR. Briefly, $100 \mathrm{ng}$ of cDNA was mixed with $10 \mu \mathrm{L} 2 \mathrm{X}$ SensiFAST SYBR No-ROX Mix, 10 $\mu \mathrm{M}$ forward primer, $10 \mu \mathrm{M}$ reverse primer. (Table 1 shows primer sequences, Integrated DNA Technologies) and nuclease-free water to $20 \mu \mathrm{L}$. A 3-step cycling was used on a Bio-Rad CFX96 instrument: 1 cycle of $95^{\circ} \mathrm{C}$ for 2 minutes to activate the polymerase, followed by 40 cycles of $95^{\circ} \mathrm{C}$ for $5 \mathrm{~s}$ to denature, $65^{\circ} \mathrm{C}$ for $10 \mathrm{~s}$ for annealing, and $10 \mathrm{~s}$ at $72^{\circ} \mathrm{C}$ for extension. Gene expression of VEGF, TNF- $\alpha$, IL-6 MRC-1, and IL-10 were normalized to GAPDH and presented as relative values.

\section{Human mesenchymal stem cell culture}

Human bone marrow-derived mesenchymal stem cells were obtained from Lonza (Walkersville, MD). Human mesenchymal stem cells (hMSCs) were cultured in a basal medium consisting of DMEM/F12 (Gibco), 10\% FBS (Atlas), and 1\% penicillin/streptomycin (Invitrogen). Medium was changed every 5 days and cells were passaged once $80 \%$ confluency was reached.

\section{Effect of resveratrol on hMSC proliferation and osteogenic differentiation}

To study the impact of resveratrol on osteogenic differentiation, hMSCs were seeded in a 24 well plate $(50,000$ cells/well) for $24 \mathrm{~h}$

\begin{tabular}{|c|c|}
\hline Gene & 5'-3' primer sequences: (F: forward R: reverse) \\
\hline \multirow[t]{2}{*}{ VEGF-A } & F: ATC TGC ATG GTG ATG TTG GA \\
\hline & R: GGG CAG AAT CAT CAC GAA GT \\
\hline \multirow[t]{2}{*}{ IL-10 } & F: GTG ATG CCC CAA GCT GAG A \\
\hline & R: CAC GGC CTT GCT CTT GTT TT \\
\hline \multirow[t]{2}{*}{ TNF- $\alpha$} & F: CTG CTG CAC TTT GGA GTG AT \\
\hline & R: AGA TGA TCT GAC TGC CTG GG \\
\hline \multirow[t]{2}{*}{ MRC-1 } & F: CAG CGC TTG TGA TCT TCA TT \\
\hline & R: TAC CCC TGC TCC TGG TTT TT \\
\hline \multirow[t]{2}{*}{ IL-6 } & F:AGC CAC TCA CCT CTT CAG AAC \\
\hline & R: GCC TCT TTG CTG CTT TCA CAC \\
\hline \multirow[t]{2}{*}{ BGLAP } & F: GGC GCT ACC TGT ATC AAT GG \\
\hline & R: TCA GCC AAC TCG TCA CAG TC \\
\hline \multirow[t]{2}{*}{ RUNX2 } & F: CTC ACT ACC ACA CCT ACC TG \\
\hline & R: TCAATA TGG TCG CCAAAC AGA TTC \\
\hline \multirow[t]{2}{*}{ GAPDH } & F: GTG GAC CTG ACC TGC CGT CT \\
\hline & R: GGA GGA GTG GGT GTC GCT GT \\
\hline
\end{tabular}

Table 1: Primers used for quantitative real-time polymerase chain reaction. 
after which the culture medium was changed. Four conditions were considered in this study: basal medium (negative control), basal medium supplemented with $12.5 \mu \mathrm{M}$ resveratrol (Sigma), osteogenic medium, or osteogenic medium supplemented with $12.5 \mu \mathrm{M}$ resveratrol. ADSCs cultured with resveratrol exhibited the highest levels of osteocalcin and osteoprotegerin at a concentration of $12.5 \mu \mathrm{M}$ [55]. Osteogenic medium consisted of DMEM F/12 (444 mL)(Invitrogen), fetal bovine serum $(50 \mathrm{~mL}), \beta$-glycerophosphate $(10 \mathrm{mM})$, ascorbic acid $(50 \mu \mathrm{g} / \mathrm{ml})($ Sigma $)$, dexamethasone $(1 \mu \mathrm{M})($ Sigma), and penicillin/ streptomyacin $(5 \mathrm{ml})$. Growth media was exchanged 3 times per week for all groups. Samples were characterized by MTS proliferation assay, osteocalcin immunofluorescence staining, Alizarin Red S staining, calcium quantification, alkaline phosphatase (ALP) staining, and ALP quantification.

Cell proliferation was monitored using [3-(4,5-dimethylthiazol-2yl)-5-(3-carboxymethoxyphenyl)-2-(4-sulfophenyl)-2H-tetrazolium (MTS) (Promega) colorimetric assay. Fresh media was added to each well at a volume of $100 \mu \mathrm{L}$, and incubated for 4 hours with $20 \mu \mathrm{L}$ of MTS solution. The resulting solution was diluted 1:5 and the absorbance was read at $492 \mathrm{~nm}$ using a Biotek Synergy 2 plate reader.

Osteocalcin was qualitatively assessed using immunofluorescence staining. Cells were fixed in $10 \%$ formalin for 30 minutes, followed by washing with a rinse buffer (2X) $(20 \mathrm{mM}$ Tris-HCL and $0.05 \%$ Tween20 in PBS) (Sigma). The samples were permeabilized with $0.1 \%$ Triton X-100 (Sigma) in PBS for 15 minutes. Cells were washed 2X with the rinse buffer, and blocked with $4 \%$ goat serum in PBS for 30 minutes. The primary antibody, osteocalcin (1:50) (R\&D Systems), was added to the samples and incubated overnight at $4^{\circ} \mathrm{C}$. Following the primary antibody incubation, the cells were washed $3 \mathrm{X}$ with the rinse buffer for 5 minutes each time. Then Alexafluor 594 (1:1000) (Invitrogen) was added to the samples and incubated for 1 hour at room temperature, followed by 4'-6-diamidino-2-phenylindole (1:25) (DAPI) (Sigma) nuclear stain. Images of stained cells were obtained using a Nikon Eclipse 80i with NIS-Elements imaging software.

To visualize the mineralized calcium, samples were fixed in $10 \%$ formalin for 30 minutes and washed $3 \mathrm{X}$ with DI water. Alizarin Red S staining solution ( $\mathrm{pH} 4.2-4.5)$ (Alfa Aesar) was added to the samples at a concentration of $0.02 \mathrm{mg} / \mathrm{mL}$ and incubated for 5 minutes. Samples were washed for 6 hours in $100 \%$ ethanol as ethanol wash solution was changed every 30 minutes. Mineralized ECM was imaged with a Nikon E600 light microscope. To quantify the mineralized calcium, the O cresolphthalein complexone (Sigma kit \#MAK022) method was employed. The scaffolds were washed with deionized (DI) water $3 \mathrm{X}$ followed by the use of $0.6 \mathrm{~mol} / \mathrm{L}$ hydrochloric acid to homogenize the samples and 4 hours of shaking at $4^{\circ} \mathrm{C}$ for total calcium extraction. Each sample was added to individual wells of a 96 well plate at a volume of $50 \mu \mathrm{L}$, and $90 \mu \mathrm{L}$ of the supplied chromogenic reagent was placed in each well containing the samples. After mixing gently, a total of $60 \mu \mathrm{L}$ of calcium assay buffer was added to each well and carefully merged with the other components. The reaction was incubated for 10 minutes in the dark, and the amount of calcium was determined by reading the absorbance at $575 \mathrm{~nm}$ with a Biotek Synergy 2 plate reader.

ALP expression was quantified using a Bio-Rad Alkaline Phosphatase Substrate Kit (\#172-1063). Briefly, cells were washed 3X with PBS, then lysed using $0.1 \%$ Triton X-100 (Sigma) in PBS for 15 minutes at $4^{\circ} \mathrm{C}$. The substrate solution was reconstituted by mixing 1 $\mathrm{mL}$ of $5 \mathrm{X}$ diethanolamine buffer with $4 \mathrm{~mL}$ DI water, then dissolving 1 Tablet of $5 \mathrm{mg}$ p-nitrophenylphosphate in the buffer mixture. Each sample was placed in individual wells of a 12 well plate at a volume of
$400 \mu \mathrm{L}$. A total of $100 \mu \mathrm{L}$ substrate solution was added to the samples and incubated at $37^{\circ} \mathrm{C}$ for 30 minutes in the dark after which $100 \mu \mathrm{L}$ $0.4 \mathrm{M} \mathrm{NaOH}$ to stop the reaction. The absorbance was read at $405 \mathrm{~nm}$ using a Biotek Synergy 2 plate reader. ALP was quantitatively detected by using alkaline phosphatase kit \#85 (Sigma) in which scaffolds were fixed with $10 \%$ formalin for 30 minutes and washed $3 \mathrm{X}$ with PBS. The Fast Blue capsule was dissolved in napthanol to prepare the staining solution, added to the scaffold, and incubated for 30 minutes. The scaffolds were washed $3 \mathrm{X}$ with PBS followed by incubation in the Mayer's Hematoxylin solution for 10 minutes. ALP was observed and imaged using a Nikon E600 light microscope

\section{Fabrication of PLGA resveratrol nanoparticles and determination of resveratrol release profile}

Nanoparticles with diameters of 150-350 nm were synthesized through a nanoprecipitation method. Two different molecular weights of poly (lactic-co-glycolic acid) (PLGA) (Lactel Absorbable Polymers) were used to fabricate the resveratrol nanoparticles in order to test individual release profiles and optimize the resveratrol dosage in the $3 \mathrm{D}$ scaffolds (Table 2). A steric solution of $2.5 \%$ poly(vinyl alcohol) (Sigma) and $0.95 \mathrm{~g}$ of 2-morpholinoethanesulfonic acid monohydrate buffer (MES) (Sigma) pH of 5.0 in $100 \mathrm{~mL}$ of deionized water was created. The polymer-resveratrol encapsulation solution was made by dissolving $100 \mathrm{mg}$ of PLGA and $2.5 \mathrm{mg}$ of resveratrol (Sigma) in 5 $\mathrm{mL}$ of acetone (Macron). The polymer-resveratrol solution was added drop-wise at a rate of $1 \mathrm{~mL} /$ minute to the steric solution while being stirred at $300 \mathrm{rpm}$. This colloidal suspension was stirred for 24 hours to ensure complete evaporation of acetone. Nanoparticles were harvested through a centrifugation step followed by lyophilization.

Transmission Electron Microscopy (TEM) was used to analyze nanoparticle size. Briefly, nanoparticles were suspended in DI water and a drop of the solution was placed on a thin carbon film. The film was placed in a vacuum to allow for water evaporation and the sample was placed on a TEM grid. Images were taken using a JEOL 200CX transmission electron microscope.

Using a UV-Vis Spectrophotometer, a calibration curve was generated to correlate the absorption at $327 \mathrm{~nm}$ (peak absorbance of resveratrol) for various concentrations. Nanoparticles with different molecular weights were incubated in $\mathrm{PBS}$ at $37^{\circ} \mathrm{C}$, and the supernatant was collected every 24 hours to read the absorbance with the UV-Vis Spectrophotometer. As it will be shown in the results, the PLGA (75:25 lactide to glycolide ratio) demonstrated the optimum release dose and was selected for use in the remainder of experiments.

\section{Synthesis of PLGA microsphere-sintered and resveratrol nanoparticle-incorporated scaffolds}

Scaffolds with diameters of $10 \mathrm{~mm}$ and heights of $2 \mathrm{~mm}$ were fabricated according to established laboratory procedures [56-58]. Briefly, PLGA (85:15 lactide to glycolide ratio) (Lactel Absorbable Polymers) was dissolved in dichloromethane (Sigma) to form a 1:4 w/v polymer solution. The solution was slowly poured into a $1 \%$ poly(vinyl alcohol) solution (Sigma) while being stirred at $330 \mathrm{rpm}$ for 24 hours to allow for adequate evaporation of the solvent. Vacuum filtration was

\begin{tabular}{|c|c|c|}
\hline Lactide to Glycolide Ratio & Molecular Weight (kDa) & Functional Group \\
\hline $75: 25$ & $75-100$ & Ester Terminated \\
\hline $50: 50$ & $16-29$ & Ester Terminated \\
\hline
\end{tabular}

Table 2: The chemical characteristics of PLGA used to design nanoparticles for resveratrol encapsulation and release in scaffolds. 
used to collect the microspheres, and the samples were washed with DI water and stored at $-20^{\circ} \mathrm{C}$ for 24 hours. The microspheres were lyophilized to completely remove all moisture. Micron sieves were used to isolate microspheres of diameter $500-700 \mu \mathrm{m}$.

Microspheres of the size range $500-700 \mu \mathrm{m}$ were placed into stainless steel molds, heated at $80^{\circ} \mathrm{C}$ for 4 hours, and sintered into cylindrical disks. Scaffolds that were to be incorporated with nanoparticles were sintered for 3.5 hours to account for the additional sintering time needed to attach nanoparticles to the microspheres.

The target resveratrol release from the nanoparticles is based on $2 \mathrm{D}$ studies demonstrating the optimum resveratrol dose for hMSC differentiation [53-55], and the desire to i) use acute inflammation to drive early osteogenesis and ii) switch macrophage phenotype to M2 after one week to promote wound healing and angiogenesis. To this end, initial resveratrol release was designed to attain 1-3 $\mu \mathrm{M}$ resveratrol for days $1-7$, and $5-12.5 \mu \mathrm{M}$ for days $7-21$ to optimize inflammation modulation and osteogenesis of hMSCs. To accomplish this, nanoparticles were suspended in DI water at a desired concentration of $180 \mu \mathrm{g}$ resveratrol nanoparticles/ $\mu \mathrm{L}$ based on the ratio of resveratrol to PLGA in the loaded nanoparticles (1:80). Next, $10 \mu \mathrm{L}$ of the nanoparticle solution was placed on each scaffold and allowed to disperse throughout the substrate. Scaffolds with nanoparticles were sintered for an additional 30 minutes at $85^{\circ} \mathrm{C}$.

Nanoparticle incorporation onto the PLGA scaffolds was confirmed using Scanning Electron Microscopy (SEM). Briefly, scaffolds were dehydrated using an ethanol sequence for 15 minutes each. Scaffolds were dried overnight in a fume hood to allow for ethanol evaporation, and coated with gold/palladium. Scaffolds were observed under Zeiss Ultra Plus FESEM after coating.

\section{Osteogenic differentiation of hMSCs as cultured on resveratrol-incorporated PLGA scaffolds}

To confirm the effect of resveratrol on osteogenic differentiation, we analyzed the growth and lineage specification of hMSCs as cultured on resveratrol-incorporated 3D PLGA scaffolds. PLGA scaffolds were first sterilized by immersion in $70 \%$ ethanol for 10 minutes. Scaffolds were washed $3 \mathrm{X}$ with PBS, and exposed to UV light for 1 hour per side to further sterilize the substrates. To seed cells on scaffolds, hMSCs were trypsinized, counted, centrifuged, and resuspended in basal medium at a concentration of 2,500 cells $/ \mu \mathrm{L}$. Cell suspension was added to each scaffold at the amount of $20 \mu \mathrm{L} /$ scaffold. Samples were incubated for 20 minutes to ensure optimum cell attachment. Next, the remaining culture medium was placed in each well containing scaffolds. Culture medium was changed to osteogenic medium after 24 hour of incubation with basal media. Cell proliferation was assessed using MTS colorimetric assay.

Osteogenic differentiation was analyzed based on the quantification of ALP and calcium expression.

\section{Inflammatory macrophage phenotype response to resveratrol as cultured on PLGA scaffolds}

THP-1 monocytes were differentiated to M1 macrophages at a seeding density of 50,000 cells/well of a 24 well plate. This was accomplished according to the protocol mentioned in the section titled "Differentiation of Monocytes to Macrophages." Resveratrol nanoparticle-incorporated scaffolds were prepared using the method described in the section "Synthesis of PLGA microsphere-sintered and resveratrol nanoparticle-incorporated scaffolds." Scaffolds were sterilized by immersion in 70\% ethanol for 10 minutes followed by rinsing $3 \mathrm{X}$ with PBS. Scaffolds were placed under UV light for 1 hour per side. Scaffolds were placed in the wells with M1 macrophages and timepoints were taken at days 3, 7, 14, and 21. Gene expression of IL-6, TNF- $\alpha$, IL-10, and VEGF was determined using qRT-PCR methods.

\section{Statistical analysis}

All experiments were done in triplicate. Three samples $(n=3)$ were analyzed per condition unless otherwise stated. Studies investigating the dose dependent effect of resveratrol on M1 macrophages, the effect of resveratrol on hMSCs, and the osteogenic differentiation of hMSCs on resveratrol incorporated PLGA were done in duplicate trials. Error bars in graphs represent mean \pm standard deviation (SD). Two-way analysis of variance (two-way ANOVA) was calculated using GraphPad Prism software and used to determine statistical significance between experimental groups. One-way analysis of variance (one-way ANOVA) with a Tukey post-test was used to calculate significance between individual groups at different time points. Statistical significance was defined as $\mathrm{p} \leq 0.05$.

\section{Results}

\section{Resveratrol modulates inflammation by inducing macrophage phenotypic switch}

Figure 1 shows the dose dependent effects of resveratrol on M1 macrophages in 2D culture. Analysis of inflammatory gene (IL-6, TNF- $\alpha$ ) and anti-inflammatory gene (VEGF, MRC-1 and IL-10) expression by qRT-PCR demonstrated that resveratrol modulates inflammation and promotes anti-inflammatory cytokine expression. Inflammatory marker IL-6 was significantly lowered with the addition of $10 \mu \mathrm{M}$ and $25 \mu \mathrm{M}$ resveratrol at day 2 , and reduced equally by all concentrations of resveratrol by day 5 (Figure 1A). Consistently, TNF- $\alpha$ was greatly reduced at the 2 day time point by $25 \mu \mathrm{M}$ of resveratrol. Day 5 results showed a dose dependent trend of lowered TNF- $\alpha$ expression, with a statistically significant reduced inflammatory cytokine level expression in the 10 and $25 \mu \mathrm{M}$ resveratrol containing group (Figure 1B). The expression of anti-inflammatory marker IL-10 significantly increased at day 2 and day 5 time points with the addition of $25 \mu \mathrm{M}$ resveratrol (Figure 1C). Similarly, VEGF expression increased with the addition of resveratrol, and day 2 expression showed statistically higher levels in the $1 \mu \mathrm{M}$ group as compared to the control (Figure 1D). By day 5 , VEGF levels were the greatest with M1 macrophages cultured with $25 \mu \mathrm{M}$, followed by $10 \mu \mathrm{M}$ and $1 \mu \mathrm{M}$. In line with these results, MRC1 levels were significantly higher for the M1 macrophages cultured with $25 \mu \mathrm{M}$ resveratrol at both time points, as compared to the control group (Figure 1E).

\section{Resveratrol drives the osteogenic differentiation of hMSCs}

Figure 2 demonstrates the effects of resveratrol on the osteogenic stimulation of hMSCs. Cells were cultured in either basal hMSC medium, basal medium supplemented with resveratrol, osteogenic differentiation medium, or osteogenic differentiation medium supplemented with resveratrol. Differentiation was analyzed over a period of 21 days. It is important to note that in all of our 2D studies, hMSCs exhibited a normal proliferation curve with no significant difference in cell growth between the study groups.

Osteogenesis was assessed based on calcium, ALP, and OCN expression. Calcium expression was analyzed at days 7, 14, and 21 (Figure 2A). At each time point, cells cultured in osteogenic medium supplemented with resveratrol produced significantly higher amounts 
Citation: Rutledge KE, Cheng Q, Jabbarzadeh E (2016) Modulation of Inflammatory Response and Induction of Bone Formation Based on Combinatorial Effects of Resveratrol. J Nanomed Nanotechnol 7: 350. doi:10.4172/2157-7439.1000350

Page 5 of 10
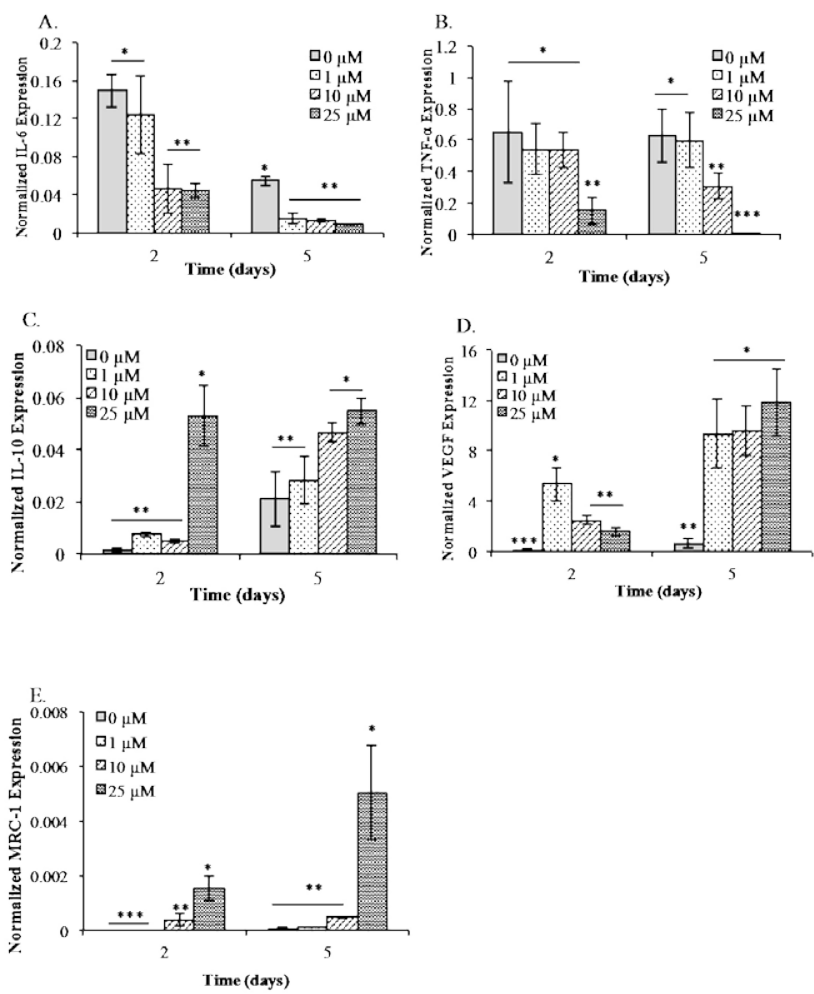

Figure 1: Resveratrol triggers the differentiation of M1 to M2 macrophages. Gene expressionby M1 macrophages cultured under different resveratrol concentration was analyzed by measuring the levels of (A) IL-6, (B) TNF- $\alpha$, (C) IL-10, (D) VEGF, and (E) MRC-1using qRT-PCR. All experiments were done in triplicate. Data points are plotted as mean $\pm S D(n=3)$. A single asterisk denotes significantly $(P \leq 0.05)$ higher as compared to groups denoted by two asterisks. Two single asterisk denotes significantly $(P \leq 0.05)$ higher as compared to groups denoted by three asterisks. Results demonstrated the highest level M2 signatory genes in the presence of resveratrol demonstrating the potential to drive the pro-angiogenic and anti-inflammatory response.

of calcium as compared to all other groups. Cells in basal medium with resveratrol as well as hMSCs cultured in osteogenic medium demonstrated a statistically higher level of calcium at days 14 , and 21 , as compared to the control. Calcium expression for hMSCs cultured in osteogenic medium with resveratrol is further confirmed by Alizarin Red S Staining (Figure 2B) showing a bright red color indicative of mineralized matrix

Alkaline Phosphatase expression was determined at 7, 14, and 21 day culture time points. Cells cultured in osteogenic medium supplemented with resveratrol exhibited a statistically higher amount of ALP at every time point as compared to all other groups (Figure 2C). Cells cultured in basal medium with resveratrol as well as osteogenic medium showed significant ALP expression at day 21. ALP staining confirmed the expression at day 21 of culture in osteogenic medium with resveratrol, as shown by the bright purple-blue color of the cells (Figure 2D).

Gene expression analysis of OCN demonstrated a significantly higher level of gene expression in the basal medium supplemented with resveratrol at day 7 (Figure 2E). At 14 and 21 day time points, cells cultured with resveratrol in osteogenic medium expressed statistically higher OCN, as compared to the control. Osteocalcin immunofluroescent staining further confirmed OCN level at day 21 expressed by hMSCs cultured in $12.5 \mu \mathrm{M}$ resveratrol (Figure $2 \mathrm{~F}$ ).

\section{Optimization of resveratrol release from the nanoparticles and incorporation in PLGA scaffolds}

Figure 3 includes the characterizations of PLGA scaffolds with resveratrol embedded nanoparticles. We observed that scaffolds possessed an interconnected porous structure consisting of microspheres sintered together. We confirmed the distribution of resveratrol nanoparticles throughout the scaffolds using SEM (Figure $3 \mathrm{~A}$ ) and TEM (Figure 3B). The images demonstrated a homogenous spreading of nanoparticles (average diameter size of $250 \mathrm{~nm}$ ) on the surface of microspheres. As expected, the nanoparticles were sintered into the PLGA scaffolds following the heating step, as manifested in the high magnification images. To tune the release of resveratrol throughout scaffolds, we varied the molecular weight and lactide/

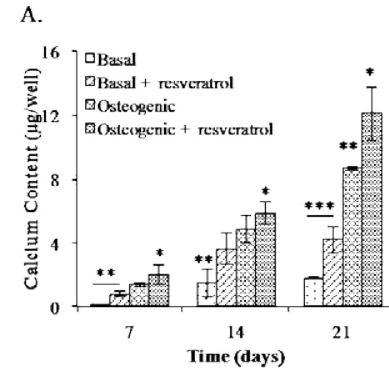

B.
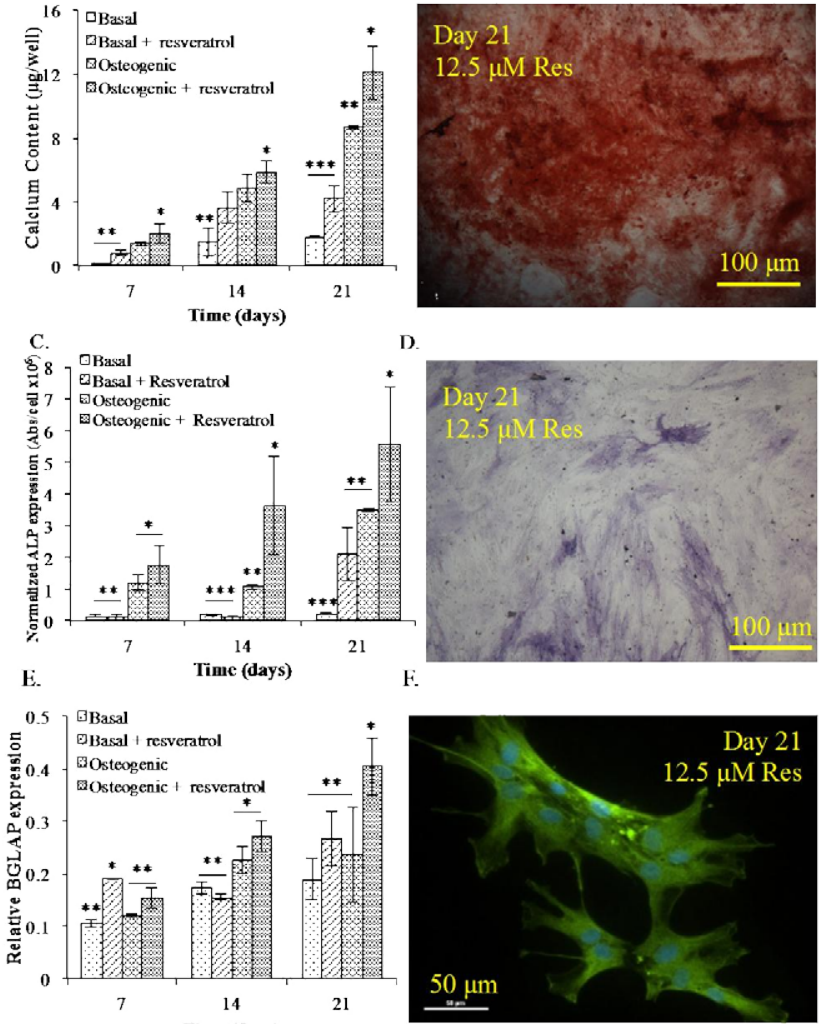

Time (days)

Figure 2: Resveratrol enhances the osteogenic differentiation of hMSCs in 2D culture. (A) Calcium expression was the greatest at all time points for hMSCs cultured in osteogenic medium in the presence of resveratrol. (B) Alizarin Red $S$ staining confirmed enhanced calcium deposition by hMSCs cultured in osteogenic medium with resveratrol at day 21 , scale bar is $100 \mu \mathrm{m}$. (C) ALP expression levels were the highest for hMSCs cultured in osteogenic medium with resveratrol for each time point, followed by osteogenic medium and basal medium supplemented with resveratrol. (D) ALP staining of hMSCs and basal medium supplemented with resveratrol. (D) ALP staining of hMSCs
at day 21 cultured in osteogenic medium with resveratrol, scale bar is 100 $\mu \mathrm{m}$. (E) qRT-PCR gene expression of hMSCs cultured in basal medium, basal media $+12.5 \mu \mathrm{M}$ resveratrol, osteogenic medium, and osteogenic medium $+12.5 \mu \mathrm{M}$ resveratrol. At days 14 and 21 , the highest level of BGLAP was detected in hMSCs cultured in osteogenic medium supplemented with resveratrol. (F) Osteocalcin staining of day $21 \mathrm{hMSCs}$ cultured in osteogenic medium $+12.5 \mu \mathrm{M}$ resveratrol, scale bar is $50 \mu \mathrm{m}$. All experiments were done in triplicate. Data points are plotted as mean \pm SD $(n=3)$. A single asterisk denotes significantly $(P \leq 0.05)$ higher as compared to groups denoted by two asterisks. Two asterisks denotes significantly $(P \leq 0.05)$ higher as compared to groups denoted by three asterisks. 

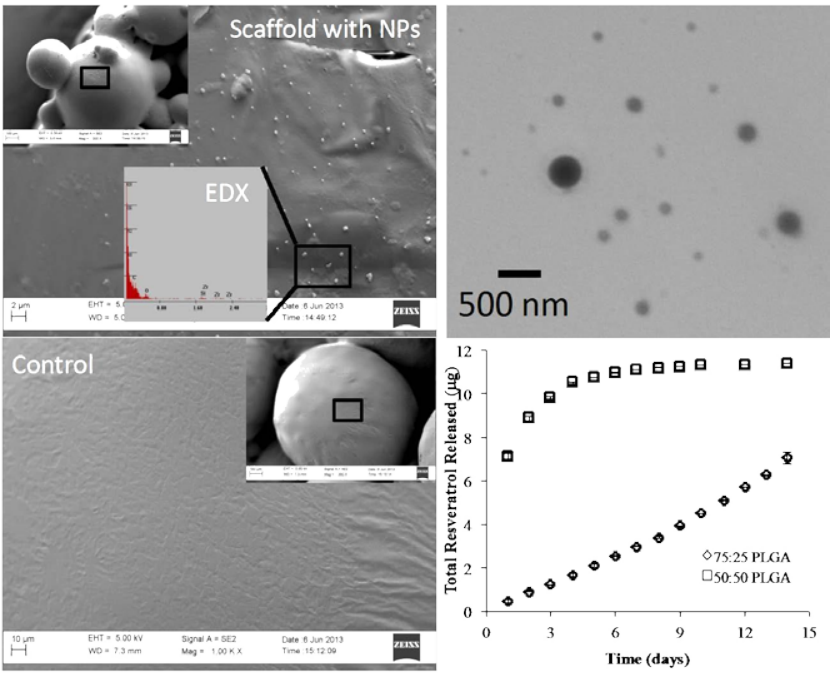

Figure 3: Scaffold characterization and resveratrol release profile. (A) SEM image of aresveratrol nanoparticle-incorporated scaffold showing the nanoparticles on the surface of the sintered microsphere scaffolds PLGA. (B) SEM image of a blank PLGA scaffold (control) demonstrating a smooth surface without nanoparticles. (C) TEM image of individual resveratrol encapsulated nanoparticles. The approximate diameter of nanoparticles was $250 \mathrm{~nm}$. (D) Resveratrol release profile from the scaffolds demonstrated a slower and more linear release when encapsulated with a higher molecular weight of PLGA.

glycolide ratio of PLGA nanoparticles. These two parameters influence the degradation of nanoparticles and the rate at which resveratrol is released in the media. Our goal was to design a sustained release of resveratrol to promote hMSC osteogenic differentiation and regulate macrophage phenotype. Figure 3D demonstrates the release profile of resveratrol from nanoparticles embedded in scaffolds. Our experimental results showed the 50/50 lactide/glycolide ratio to cause a burst release of resveratrol followed by a plateau region. This is undesirable as we needed cells to be exposed to a controlled dose of resveratrol over a long period. Thus, we chose the higher lactide/glycolide ratio of 75/25 for the rest of this study to avoid burst release. In addition, we chose a higher molecular weight of $75-100 \mathrm{kDa}$ to prolong the resveratrol release profile within the 3D PLGA sintered microsphere scaffolds.

\section{Resveratrol nanoparticle-incorporated scaffolds mediate macrophage polarization and enhance osteogenic differentiation of hMSCs}

M1 macrophages were cultured on resveratrol incorporated PLGA scaffolds for 21 days, and inflammatory gene expression was analyzed at different time points (Figure 4). We observed the expression of IL-6 xpression to sustain in day 3 to day 7 time points, and significantly drop by day 14 (Figure 4A). Consistently, the gene expression of inflammatory marker TNF- $\alpha$ was significantly lower on later time points (Figure $4 \mathrm{~B}$ ). On the other hand, IL-10 and VEGF expression were upregulated at the day 21 time point (Figures $4 \mathrm{C}$ and $4 \mathrm{D}$ ). These results were consistent with our $2 \mathrm{D}$ experiments in that resveratrol downregulates the inflammatory markers while stimulating the expression of angiogenic genes. A difference, however, is manifested in the amounts of these genes due to the $3 \mathrm{D}$ nature of scaffolds and the release profile of resveratrol on scaffolds versus $2 \mathrm{D}$ conditions.

Figure 5 demonstrates the results of hMSCs cultured on tissue culture polystyrene (TCPS), 3D PLGA scaffolds, and 3D resveratrol nanoparticle-incorporated PLGA scaffolds. We observed that the cells grow in all conditions; however, hMSCs cultured on TCPS medium had significantly higher cell numbers than both the PLGA and PLGA with resveratrol groups (Figure 5A). Furthermore, the cells cultured on blank PLGA reached a significantly higher number compared to hMSCs grown on PLGA with resveratrol nanoparticles.

hMSCs cultured on resveratrol- incorporated scaffolds demonstrated significantly greater of ALP expression compared to all other experimental groups at 7, 14, and 21 day time points, while hMSCs on PLGA scaffolds produced the second highest amount of ALP at day 21 (Figure 5B). Consistently, hMSCs cultured on resveratrol nanoparticle scaffolds expressed a significantly higher amount of calcium at days 7, 14 and 21 (Figure 5C). These results were consistent with the 2D experiments demonstrating the potential of resveratrol to promote osteogenic differentiation of stem cells.

\section{Discussion}

Our study addresses fundamental issues facing osseointegration of biomaterial grafts in an inflammatory microenvironment. We set out to engineer an immunomodulatory and osteoinductive scaffold that can harness the osteogenic and wound healing potential of immune cells, as well as program hMSCs towards bone tissue formation. To accomplish this goal, we demonstrated the ability of resveratrol to mediate macrophage phenotype from inflammatory to wound healing as well as stimulate osteogenic differentiation of hMSCs. Building on these $2 \mathrm{D}$ and $3 \mathrm{D}$ proof-of-concept experiments, we designed a specific resveratrol nanoparticle release profile within PLGA scaffolds that would i) mediate macrophage phenotype and subsequent cytokine
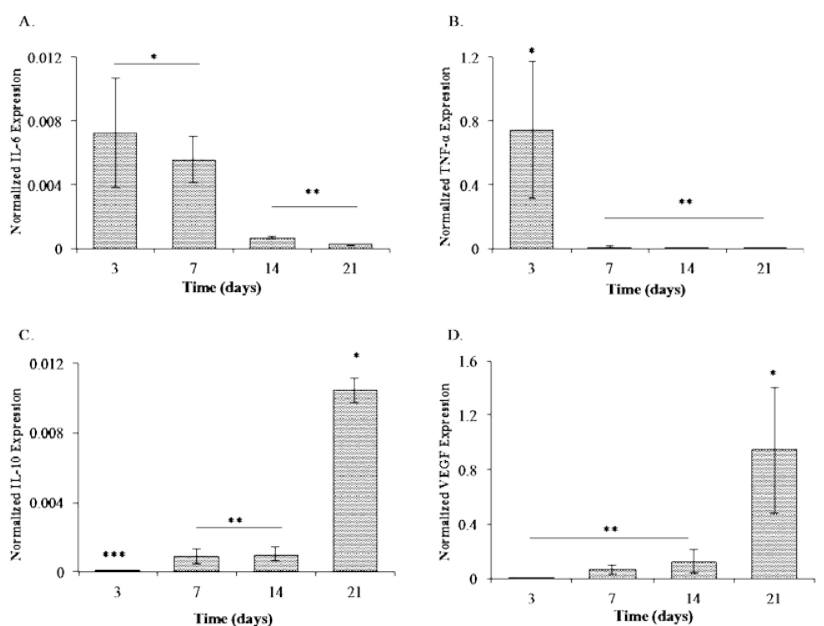

Figure 4: Resveratrol incorporated PLGA scaffolds ameliorated inflammatory genes and upregulated wound healing markers. Gene expression analysis of M1 macrophages on theresveratrol incorporated scaffolds demonstrated (A) IL-6 expression at days 3 to 7, 14 and 21 post-culture. (B) TNF- $\alpha$ expression at days 3 to 7,14 and 21 post-culture. A single asterisk denotes a significantly higher expression of TNF- $\alpha$ at day 3 compared to the previous time point. (C) IL-10 expression at days 3 to 7,14 and 21 post-culture. (D) VEGF expression at days 3 to 7,14 and 21 post-culture. A single asterisk denotes a significantly higher expression of VEGF at day 21. All experiments were done in triplicate. Data points are plotted as mean $\pm S D(n=3)$. A single asterisk denotes significantly $(P \leq 0.05)$ higher as compared to groups denoted by two asterisks. Two asterisks denotes significantly $(P \leq 0.05)$ higher as compared to groups denoted by three asterisks. Results showed the highest level M2 signatory genes in the presence of resveratrol demonstrating the potential to drive the pro-angiogenic anti-inflammatory response. 

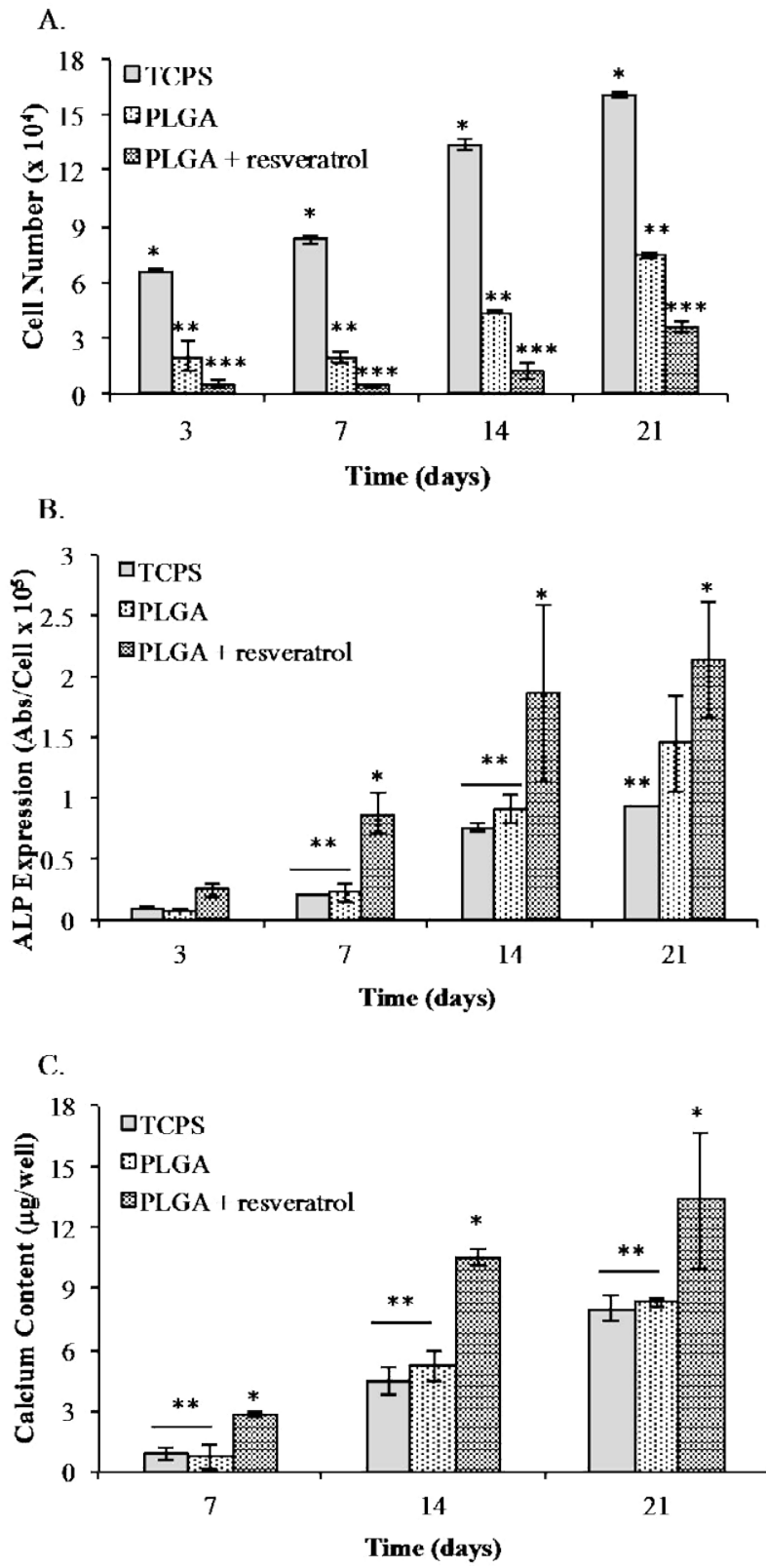

Figure 5: Resveratrol enhanced the osteogenic differentiation of hMSCs on PLGA scaffold. (A)hMSC proliferation on the scaffolds showed a normal cell growth curve with the highest cell numbers shown for the TCPS group followed by the PLGA and PLGA scaffolds with resveratrol. (B) ALP expression by hMSCs on TCPS, PLGA scaffolds and PLGA scaffolds with resveratrol nanoparticles at various time points. (C) ALP expression by hMSCs on TCPS, PLGA scaffolds and PLGA scaffolds with resveratrol nanoparticles at various time points. All experiments were done in triplicate. Data points are plotted as mean $\pm S D(n=3)$. A single asterisk denotes significantly $(P \leq 0.05)$ higher as compared to groups denoted by two asterisks. Two asterisks denotes significantly $(P \leq 0.05)$ higher as compared to groups denoted by three asterisks.

secretion and ii) drive the osteogenic differentiation of hMSCs. This resveratrol nanoparticle incorporated scaffold is a novel approach to enhance graft integration and assimilation with native tissue.

Researchers have explored many options to modulate the immune response to prevent biomaterial rejection, such as using polymeric coatings, steroidal anti-inflammatory drugs, and angiogenic factors.
These methods have faced many limitations due to immunogenicity, decomposition during the manufacturing process, and poor adhesion characteristics. Anti-inflammatory pharmaceutical drugs only temporary suppress inflammation and have been known to reduce angiogenic factors which in turn delays wound healing. Finally, despite their important role in inflammatory cascade of events, the use of angiogenic growth factors is only partially effective due to its physiological side effects.

Instead of trying to counteract inflammation, our approach aims to utilize a natural polyphenol to regulate immune cell behavior and use their expressed signaling molecules to drive the osteogenic differentiation of hMSCs. Our methods build on the central concepts of the anti-inflammatory and osteogenic properties of resveratrol as well as the known angiogenic signaling molecules produced by macrophages. To this end, we fabricated resveratrol nanoparticleincorporated scaffolds with a controlled release profile that have the potential to revolutionize biomaterial assimilation with native bone tissue.

Our proof of concept experiments investigating the dose dependent regulation of inflammatory cytokines by resveratrol indicated that M1 macrophages produce less IL- 6 and TNF- $\alpha$ when cultured with the anti-inflammatory polyphenol. This is coupled with higher expression of anti-inflammatory markers VEGF, IL-10, and MRC-1 after 48 hours of exposure to resveratrol. Cells cultured with $25 \mu \mathrm{M}$ resveratrol demonstrated the greatest expression of wound healing markers VEGF, MRC-1 and IL-10. Consistently, M1 macrophages cultured with 25 $\mu \mathrm{M}$ resveratrol exhibited the lowest amount of inflammatory cytokine TNF- $\alpha$. Inflammatory marker IL- 6 reduction by resveratrol was not statistically different between the $10 \mu \mathrm{M}$ and $25 \mu \mathrm{M}$ groups. From these results, it is clear that $25 \mu \mathrm{M}$ resveratrol modulates inflammation to the greatest extent as compared to the other groups. However, the optimized time scale and amount of resveratrol released from the nanoparticles should account for the target temporal concentration for both osteogenesis and macrophage phenotype polarization.

The use of resveratrol as a stimulator of osteogenesis has been previously demonstrated for hMSCs, human adipose derived stem cells (hADSCs), and pre-osteoblastic MC3T3-E1 cells [55]. Furthermore, studies have shown resveratrol to affect proliferation and osteogenesis in a dose-dependent manner. hADSCs cultured with $12.5 \mu \mathrm{M}, 25 \mu \mathrm{M}$, and $50 \mu \mathrm{M}$ resveratrol showed the highest proliferation rate when exposed to $12.5 \mu \mathrm{M}$ resveratrol, and the highest levels of ALP when cultured with $25 \mu \mathrm{M}$ resveratrol. Doses of $50 \mu \mathrm{M}$ resulted in extremely low cell numbers and ALP production. ADSCs cultured with resveratrol exhibited the highest levels of osteocalcin and osteoprotegerin at a concentration of $12.5 \mu \mathrm{M}$ [55]. Another study concluded that doses higher than $25 \mu \mathrm{M}$ resveratrol are potentially cytotoxic, and that $12.5 \mu \mathrm{M}$ resveratrol results in the greatest mineralized matrix after 4 weeks in vivo [53]. Additionally, hMSCs cultured with varying doses of resveratrol produced the highest calcium deposition and greatest proliferative capabilities when exposed to a concentration of $10 \mu \mathrm{M}$ [5963]. Based on these studies, and the fact that M1 macrophages switch phenotype to M2 when exposed to doses as low as $1 \mu \mathrm{M}$ resveratrol, we selected a target resveratrol concentration of $12.5 \mu \mathrm{M}$ to stimulate osteogenesis of hMSCs in 2D. Consistent with previous studies, we observed the greatest calcium deposition and ALP expression from cells cultured in osteogenic medium $+12.5 \mu \mathrm{M}$. Furthermore, OCN expression level was the highest for hMSCs cultured in osteogenic medium $+12.5 \mu \mathrm{M}$. To optimize macrophage control and osteogenic differentiation of hMSCs, we targeted a nanoparticle release profile 
of approximately 1-3 $\mu \mathrm{M}$ resveratrol per day for days $1-7$, then approximately $5-12.5 \mu \mathrm{M}$ resveratrol per day for days 7-21.

To design a biomaterial that allows for modulation of the immune response, one must first determine how specific aspects of inflammation such as macrophage phenotype influence wound healing and osteogenesis. Preliminary investigations on total joint replacement and the surrounding tissue histology from either i) joints that had become loose due to osteolysis, and ii) joints implanted in osteoarthritic patients, have found that the former tissue produced many M1 macrophages while the latter demonstrated M2 macrophages $[64,65]$. In another recent study, porosity was found to drive the higher ratio of M2/M1 macrophages when compared to the non-porous control [66]. Furthermore, scaffolds composed of natural ECM can switch macrophage phenotype to predominantly wound healing by $7-14$ days after implantation [67-69]. The common thread that relates all these findings is that they all rely on altering the cytokine release profile by monocyte and macrophages to attenuate the inflammatory response to the biomaterial [70,71].

Although chronic inflammation is detrimental to wound healing and assimilation of graft with native tissue, studies have demonstrated the benefits of monocytes and macrophages in stimulating osteogenic differentiation of stem cells. In a recent published work, hMSCs were cultured in conditioned medium (CM) from M1 macrophages, M2 macrophages, and monocytes, and analyzed for hallmark osteogenic markers such as RUNX2, ALP, and bone morphogenetic protein-2 (BMP-2). hMSCs cultured with M1 CM expressed the highest levels of RUNX2, ALP, and BMP-2 [72]. Another study demonstrated that a member of the IL-6 pro-inflammatory cytokine family, Oncostatin M (OSM), produced by M1 macrophages promoted osteogenic differentiation of hMSCs and inhibited adipogenesis [73]. Macrophages secrete several osteogenic signaling molecules such as bone morphogenic protein-2 (BMP-2), 1, 25-dihydroxyvitamin D3, interleukin-1 beta (IL-1 $\beta$ ), and IL-6 [74-76]. During fracture healing, cytokine members of the TGF- $\beta$ superfamily, such as BMP, promote different stages of wound repair. BMP-2 peaks in expression levels early in the healing process, mediates a cascade of other BMPs associated with intramembranous and endochondral ossification [77]. TNF- $\alpha$ is another cytokine secreted by macrophages during the initial inflammatory response that is responsible for recruiting hMSCs, and promoting cell survival [78]. Additionally, macrophages secrete angiogenic growth factors such as VEGF and PDGF, and these cytokines are important mediators in bone remodeling. Specifically, the VEGF family recruits endothelial cells, osteoblasts, and osteoclasts, and can promote microvascular endothelial cells to secrete BMPs in a hypoxic microenvironment found in fractured bone tissue [79-81].

In our proof of concept 3D experiments, we were able to successfully engineer a scaffold with a specific resveratrol release profile. M1 macrophages and hMSCs were individually placed on the scaffolds and cultured for 21 days. The gene expression profile of the macrophages showed moderately high expression of IL- 6 for day 3 and day 7 , and was significantly reduced by day 14 . VEGF expression levels were relatively low until day 14, but significantly increased by day 21 . Our method of inducing endogenous VEGF physiologic secretion from native macrophages provides a potential avenue to enhance vascular infiltration in bone scaffolds.

Stem cells cultured on the resveratrol-incorporated scaffolds expressed the highest levels of calcium and ALP, demonstrating the effectiveness of the controlled resveratrol release. The mechanism by which resveratrol induces osteogenesis is under deep investigation.
Preliminary work by scientists has shown resveratrol to trigger Wnt signaling pathway leading to the upregulation of RUNX2 expression, the transcription factor essential for cell differentiation into osteoblasts $[51,82]$. Resveratrol also promotes osteogenesis through SIRT-1, and it has been shown that FOXO3A protein expression and SIRT-1 activation operate synergistically to mediate RUNX2 gene transcription $[52,62]$. When embryonic stem cell-derived mesenchymal progenitors are cultured in adipogenic medium containing resveratrol, RUNX2 and OCN are upregulated while adipogenic genes PPAR $\gamma 2$ and LEPTIN are suppressed [62]. This is extremely important because PPAR $\gamma 2$ can prevent RUNX2 transcription and inhibit osteogenesis [83,84]. Further studies are warranted to illucidate the temporal effect of resveratrol of osteogenic signaling pathways using appropriate in vitro and in vivo models.

This study paves the way toward harnessing the inflammatory response to biomaterials and increases the likelihood of graft integration and based on the use of a single natural dietary molecule. Further studies are typo to transfer the findings of this study in the clinic. One major issue to be addressed is the scale up of the resveratrol release profile in an in vivo model. In general, the rate at which drugs clear in the human body follows different kinetics as compared to controlled in vitro experiments. The morphological structure of the target tissue, density of vascular networks in the vicinity of the implant, and physiologic conditions are among many factors that affect the bioavailability and release profile. In light of the encouraging in vitro results, in vivo studies should recalibrate the release kinetics based on the pharmacological and metabolic effects of resveratrol. Another limitation of this study is apparent in its simplification of an inflammatory microenvironment surrounding a biomaterial. The complex interactions of different cell types and signaling molecules in this process is overlooked in this study to establish the proof of concept. However, clinical studies should take in to account these interactions in evaluating the efficacy of this strategy in vivo. Finally, most studies to date on the hermetic behavior of resveratrol have focused on the oral consumption. Despite the positive advances, the definite test of resveratrol's pharmacokinetics and toxic effects, as released directly in the tissue, will elucidate the mechanisms of action and potential use in tissue engineering and regenerative medicine.

\section{Acknowledgement}

We gratefully acknowledge support from the National Institutes of Health grants (NIH P20 GM103641) and (NIH AR063338), and National Science Foundation grant (EPS-0903795).

\section{References}

1. Langer R, Vacanti JP (1993) Tissue engineering. Science 260: 920-926.

2. Harris GM, Rutledge K, Cheng Q, Blanchette J, Jabbarzadeh E (2013) Strategies to direct angiogenesis within scaffolds for bone tissue engineering Curr Pharm Des 19: 3456-3465.

3. Anderson JM (2001) Biological responses to materials. Ann Rev Mater Res 31: 81-110.

4. Wynn TA, Barron L (2010) Macrophages: master regulators of inflammation and fibrosis. Semin Liver Dis 30: 245-257.

5. Sindrilaru A, Peters T, Wieschalka S, Baican C, Baican A, et al. (2011) An unrestrained proinflammatory M1 macrophage population induced by iron impairs wound healing in humans and mice. J Clin Invest 121: 985-997.

6. Williams GT, Williams WJ (1983) Granulomatous inflammation- A review. Journal of clinical pathology 36: 723-733.

7. Johnston RB Jr (1988) Current concepts: immunology. Monocytes and macrophages. N Engl J Med 318: 747-752. 
Citation: Rutledge KE, Cheng Q, Jabbarzadeh E (2016) Modulation of Inflammatory Response and Induction of Bone Formation Based on Combinatorial Effects of Resveratrol. J Nanomed Nanotechnol 7: 350. doi:10.4172/2157-7439.1000350

8. Cotran RZ, Robbins SL (1999) (6th edn.) Saunders, Philadelphia, 50-112.

9. Gallin JI, SR (1999) Inflammation: Basic Principles and Clinical Correlates. (2nd edition), Raven, New York.

10. Chambers TJ, Spector WG (1982) Inflammatory giant cells. Immunobiology 161: 283-289.

11. Rae T (1986) The Macrophage Response to Implant Materials - with Specia Reference to Those Used in Orthopedics. Crit Rev Biocompat 2: 97-126.

12. Anderson JM (1988) Inflammatory response to implants. ASAIO Trans 34: 101-107.

13. Greisler H (1988) Macrophage-biomaterial interactions with bioresorbable vascular prostheses. ASAIO transactions/American Society for Artificial Internal Organs 34: 1051-1059.

14. Anderson JM (1993) Mechanisms of Inflammation and Infection with Implanted Devices. Cardiovasc Pathol 2: 33-41.

15. Das A, Segar CE, Hughley BB, Bowers DT, Botchwey EA (2013) The promotion of mandibular defect healing by the targeting of S1P receptors and the recruitment of alternatively activated macrophages. Biomaterials 34: 9853-9862.

16. Kim YH, Furuya H, Tabata Y (2014) Enhancement of bone regeneration by dual release of a macrophage recruitment agent and platelet-rich plasma from gelatin hydrogels. Biomaterials 35: 214-224.

17. Spiller KL, Nassiri S, Witherel CE, Anfang RR, Ng J, et al. (2015) Sequential delivery of immunomodulatory cytokines to facilitate the M1-to-M2 transition of macrophages and enhance vascularization of bone scaffolds. Biomaterials 37: 194-207

18. Spiller KL, Anfang RR, Spiller KJ, Ng J, Nakazawa KR, et al. (2014) The role of macrophage phenotype in vascularization of tissue engineering scaffolds. Biomaterials 35: 4477-4488.

19. Göpferich A (1996) Mechanisms of polymer degradation and erosion Biomaterials 17: 103-114.

20. Morais JM, Papadimitrakopoulos F, Burgess DJ (2010) Biomaterials/tissue interactions: possible solutions to overcome foreign body response. AAPS J 12: 188-196.

21. Shastri VP (2003) Non-degradable biocompatible polymers in medicine: past present and future. Curr Pharm Biotechnol 4: 331-337.

22. Shen M, Horbett TA (2001) The effects of surface chemistry and adsorbed proteins on monocyte/macrophage adhesion to chemically modified polystyrene surfaces. Journal of biomedical materials research 57: 336-345

23. Dalsin JL, Hu BH, Lee BP, Messersmith PB (2003) Mussel adhesive protein mimetic polymers for the preparation of nonfouling surfaces. J Am Chem Soc 125: 4253-4258.

24. Perretti M, Ahluwalia A (2000) The microcirculation and inflammation: site of action for glucocorticoids. Microcirculation 7: 147-161.

25. Morais JM, Papadimitrakopoulos F, Burgess DJ (2010) Biomaterials/tissue interactions: possible solutions to overcome foreign body response. AAPS 12: $188-196$

26. Halaby IA, Lyden SP, Davies MG, Roztocil E, Salamone LJ, et al. (2002) Glucocorticoid-regulated VEGF expression in ischemic skeletal muscle. Mo Ther 5: 300-306.

27. Gaytán F, Morales C, Bellido C, Sánchez-Criado JE (2002) Selective apoptosis of luteal endothelial cells in dexamethasone-treated rats leads to ischemic necrosis of luteal tissue. Biol Reprod 66: 232-240.

28. Ward WK (2003) Vascularizing the tissue surrounding a model biosensor: how localized is the effect of a subcutaneous infusion of vascular endothelial growth factor (VEGF)? Biosensors \& bioelectronics 19: 155-163.

29. Kedem A, Perets A, Gamlieli-Bonshtein I, Dvir-Ginzberg M, Mizrahi S, et al. (2005) Vascular endothelial growth factor-releasing scaffolds enhance vascularization and engraftment of hepatocytes transplanted on liver lobes. Tissue Eng 11: 715-722.

30. Patil SD, Papadmitrakopoulos F, Burgess DJ (2007) Concurrent delivery of dexamethasone and VEGF for localized inflammation control and angiogenesis. J Control Release 117: 68-79.

31. Henry TD (1999) Therapeutic angiogenesis. BMJ 318: 1536-1539.
32. Epstein SE, Fuchs S, Zhou YF, Baffour R, Kornowski R (2001) Therapeutic interventions for enhancing collateral development by administration of growth factors: basic principles, early results and potential hazards. Cardiovascular research 49: 532-542.

33. Emanueli C, Madeddu P (2001) Angiogenesis gene therapy to rescue ischaemic tissues: achievements and future directions. Br J Pharmacol 133: 951-958.

34. Frémont $L$ (2000) Biological effects of resveratrol. Life Sci 66: 663-673.

35. Singh G, Pai RS (2014) Optimized PLGA nanoparticle platform for orally dosed trans-resveratrol with enhanced bioavailability potential. Expert opinion on drug delivery 1: 647-659.

36. Jang M, Cai L, Udeani GO, Slowing KV, Thomas CF, et al. (1997) Cancer chemopreventive activity of resveratrol, a natural product derived from grapes. Science 275: 218-220.

37. Bradamante S, Barenghi L, Villa A (2004) Cardiovascular protective effects of resveratrol. Cardiovasc Drug Rev 22: 169-188.

38. Sinha K, Chaudhary G, Gupta YK (2002) Protective effect of resveratrol against oxidative stress in middle cerebral artery occlusion model of stroke in rats. Life Sci 71: 655-665.

39. Wang Q, Xu J, Rottinghaus GE, Simonyi A, Lubahn D, et al. (2002) Resveratrol protects against global cerebral ischemic injury in gerbils. Brain Res 958: 439-447.

40. Wu SL, Pan CE, Yu L, Meng KW (2005) Immunosuppression by combined use of cyclosporine and resveratrol in a rat liver transplantation model. Transplant Proc 37: 2354-2359.

41. Wu SL, Yu L, Meng KW, Ma ZH, Pan CE (2005) Resveratrol prolongs allograft survival after liver transplantation in rats. World J Gastroenterol 11: 4745-4749.

42. Sun AY, Wang Q, Simonyi A, Sun GY (2011) Herbal Medicine: Biomolecular and Clinical Aspects ( $2^{\text {nd }}$ edn.). In: I.F.F. Benzie \& S. Wachtel-Galor (eds.)

43. Baur JA, Pearson KJ, Price NL, Jamieson HA, Lerin C, et al. (2006) Resveratrol improves health and survival of mice on a high-calorie diet. Nature 444: 337-342.

44. Aggarwal BB, Bhardwaj A, Aggarwal RS, Seeram NP, Shishodia S, et al. (2004) Role of resveratrol in prevention and therapy of cancer: preclinical and clinical studies. Anticancer Res 24: 2783-2840.

45. Yan Y, Gao YY, Liu BQ, Niu XF, Zhuang Y, et al. (2010) Resveratrol-induced cytotoxicity in human Burkitt's lymphoma cells is coupled to the unfolded protein response. BMC Cancer 10: 445

46. Campagna M, Rivas C (2010) Antiviral activity of resveratrol. Biochem Soc Trans 38: 50-53

47. Tsai SH, Lin-Shiau SY, Lin JK (1999) Suppression of nitric oxide synthase and the down-regulation of the activation of NFkappaB in macrophages by resveratrol. Br J Pharmacol 126: 673-680.

48. Leiro J, Alvarez E, Arranz JA, Laguna R, Uriarte E, et al. (2004) Effects of cis-resveratrol on inflammatory murine macrophages: antioxidant activity and down-regulation of inflammatory genes. J Leukoc Biol 75: 1156-1165.

49. Chung EY (2011) Resveratrol down-regulates interferon-gamma-inducible inflammatory genes in macrophages: molecular mechanism via decreased STAT-1 activation. The Journal of nutritional biochemistry 22: 902-909.

50. Wang W, Sun L, Zhang P, Song J, Liu W (2014) An anti-inflammatory cell-free collagen/resveratrol scaffold for repairing osteochondral defects in rabbits. Acta Biomater 10: 4983-4995.

51. Zhou H (2009) Resveratrol augments the canonical Wnt signaling pathway in promoting osteoblastic differentiation of multipotent mesenchymal cells. Experimental cell research 315: 2953-2962.

52. Shakibaei M, Shayan P, Busch F, Aldinger C, Buhrmann C, et al. (2012) Resveratrol mediated modulation of Sirt-1/Runx2 promotes osteogenic differentiation of mesenchymal stem cells: potential role of Runx2 deacetylation. PLoS One 7: e35712.

53. Dosier CR (2012) Resveratrol effect on osteogenic differentiation of rat and human adipose derived stem cells in a 3-D culture environment. Journal of the mechanical behavior of biomedical materials 1:112-122.

54. Mizutani K, Ikeda K, Kawai Y, Yamori Y (1998) Resveratrol stimulates the proliferation and differentiation of osteoblastic MC3T3-E1 cells. Biochem Biophys Res Commun 253: 859-863. 
Citation: Rutledge KE, Cheng Q, Jabbarzadeh E (2016) Modulation of Inflammatory Response and Induction of Bone Formation Based on Combinatorial Effects of Resveratrol. J Nanomed Nanotechnol 7: 350. doi:10.4172/2157-7439.1000350

55. Erdman CP, Dosier CR, Olivares-Navarrete R, Baile C, Guldberg RE, et al (2012) Effects of resveratrol on enrichment of adipose-derived stem cells and their differentiation to osteoblasts in two-and three-dimensional cultures. $J$ Tissue Eng Regen Med 6 Suppl 3: s34-46.

56. Jabbarzadeh E, Deng M, Lv Q, Jiang T, Khan YM, et al. (2012) VEGFincorporated biomimetic poly(lactide-co-glycolide) sintered microsphere scaffolds for bone tissue engineering. J Biomed Mater Res B Appl Biomater 100: 2187-2196.

57. Jiang T, Nukavarapu SP, Deng M, Jabbarzadeh E, Kofron MD, et al. (2010) Chitosan-poly(lactide-co-glycolide) microsphere-based scaffolds for bone tissue engineering: in vitro degradation and in vivo bone regeneration studies. Acta Biomater 6: 3457-3470.

58. Rutledge K, Cheng Q, Pryzhkova M, Harris GM, Jabbarzadeh E (2014) Enhanced differentiation of human embryonic stem cells on extracellular matrix-containing osteomimetic scaffolds for bone tissue engineering. Tissue Eng Part C Methods 20: 865-874.

59. Mobasheri A, Shakibaei M (2013) Osteogenic effects of resveratrol in vitro: potential for the prevention and treatment of osteoporosis. Ann N Y Acad Sci 1290: 59-66.

60. Boissy P (2005) Resveratrol inhibits myeloma cell growth, prevents osteoclas formation, and promotes osteoblast differentiation. Cancer research 65: 99439952

61. Cdos CS, Rohden F, Hammes TO, Margis R, Bortolotto JW, et al. (2011) Resveratrol upregulated SIRT, FOXO, and adiponectin and downregulated PPAR ${ }^{3} 1-3$ mRNA expression in human visceral adipocytes. Obes Surg 21: 356-361.

62. Tseng PC, Hou SM, Chen RJ, Peng HW, Hsieh CF, et al. (2011) Resveratrol promotes osteogenesis of human mesenchymal stem cells by upregulating RUNX2 gene expression via the SIRT1/FOXO3A axis. J Bone Miner Res 26 2552-2563.

63. Dai Z (2007) Resveratrol enhances proliferation and osteoblastic differentiation in human mesenchymal stem cells via ER-dependent ERK1/2 activation. Phytomedicine: International journal of phytotherapy and phytopharmacology 14: 806-814.

64. Yamashita S, Saito Y, Jong (2013) Carbon Nanotubes and Graphene for Photonic Applications. Woodhead publishing limited.

65. Brown BN, Ratner BD, Goodman SB, Amar S, Badylak SF (2012) Macrophage polarization: an opportunity for improved outcomes in biomaterials and regenerative medicine. Biomaterials 33: 3792-3802.

66. Madden LR, Mortisen DJ, Sussman EM, Dupras SK, Fugate JA, et al. (2010) Proangiogenic scaffolds as functional templates for cardiac tissue engineering Proc Natl Acad Sci U S A 107: 15211-15216.

67. Badylak SF, Valentin JE, Ravindra AK, McCabe GP, Stewart-Akers AM (2008) Macrophage phenotype as a determinant of biologic scaffold remodeling. Tissue Eng Part A 14: 1835-1842.

68. Brown BN, Londono R, Tottey S, Zhang L, Kukla KA, et al. (2012) Macrophage phenotype as a predictor of constructive remodeling following the implantation of biologically derived surgical mesh materials. Acta Biomater 8: 978-987.

69. Brown BN, Valentin JE, Stewart-Akers AM, McCabe GP, Badylak SF (2009) Macrophage phenotype and remodeling outcomes in response to biologic scaffolds with and without a cellular component. Biomaterials 30: 1482-1491.

70. Gretzer C, Eriksson AS, Alldén B, Ericson LE, Thomsen P (1996) Monocyte activation on titanium-sputtered polystyrene surfaces in vitro: the effect of culture conditions on interleukin-1 release. Biomaterials 17: 851-858.

71. Thomsen P, Gretzer C (2001) Macrophage interactions with modified materia surfaces. Curr Opin Solid St M 5: 163-176.
72. Omar OM, Granéli C, Ekström K, Karlsson C, Johansson A, et al. (2011) The stimulation of an osteogenic response by classical monocyte activation. Biomaterials 32: 8190-8204.

73. Guihard P, Danger Y, Brounais B, David E, Brion R, et al. (2012) Induction of osteogenesis in mesenchymal stem cells by activated monocytes/macrophages depends on oncostatin M signaling. Stem Cells 30: 762-772.

74. Kreutz M (1993) 1,25-dihydroxyvitamin D3 production and vitamin D3 receptor expression are developmentally regulated during differentiation of human monocytes into macrophages. Blood 82: 1300-1307.

75. Champagne CM, Takebe J, Offenbacher S, Cooper LF (2002) Macrophage cell lines produce osteoinductive signals that include bone morphogenetic protein-2. Bone 30: 26-31.

76. Kimble RB, Bain S, Pacifici R (1997) The functional block of TNF but not of IL-6 prevents bone loss in ovariectomized mice. J Bone Miner Res 12: 935-941.

77. Cho TJ, Gerstenfeld LC, Einhorn TA (2002) Differential temporal expression of members of the transforming growth factor beta superfamily during murine fracture healing. J Bone Miner Res 17: 513-520.

78. Gerstenfeld LC, Edgar CM, Kakar S, Jacobsen KA, Einhorn TA (2007) Engineering of functional skeletal tissues.

79. Deckers MM, Karperien M, van der Bent C, Yamashita T, Papapoulos SE, et al. (2000) Expression of vascular endothelial growth factors and their receptors during osteoblast differentiation. Endocrinology 141: 1667-1674.

80. Bouletreau PJ, Warren SM, Spector JA, Peled ZM, Gerrets RP, et al. (2002) Hypoxia and VEGF up-regulate BMP-2 mRNA and protein expression in microvascular endothelial cells: implications for fracture healing. Plast Reconstr Surg 109: 2384-2397.

81. Mayr-Wohlfart U, Waltenberger J, Hausser H, Kessler S, Günther KP, et al. (2002) Vascular endothelial growth factor stimulates chemotactic migration of primary human osteoblasts. Bone 30: 472-477.

82. Gaur T, Lengner CJ, Hovhannisyan H, Bhat RA, Bodine PV, et al. (2005) Canonical WNT signaling promotes osteogenesis by directly stimulating Runx2 gene expression. J Biol Chem 280: 33132-33140.

83. Jeon MJ, Kim JA, Kwon SH, Kim SW, Park KS, et al. (2003) Activation of peroxisome proliferator-activated receptor-gamma inhibits the Runx2-mediated transcription of osteocalcin in osteoblasts. J Biol Chem 278: 23270-23277.

84. Shockley KR (2009) PPARgamma2 nuclear receptor controls multiple regulatory pathways of osteoblast differentiation from marrow mesenchyma stem cells. J Cell Biochem 106: 232-246. 